\title{
Procesos Termodinámicos
}

\section{Thermodynamic processes}

\author{
Oscar D. Ramírez-Cárdenas ${ }^{a}$, José F. Vázquez-Montiel ${ }^{b}$,Brando J. Martínez-Ramírez ${ }^{c}$, Víctor \\ D. Rodríguez-Caballero ${ }^{d}$
}

\begin{abstract}
:
A thermodynamic process is the changes of a system, from some initial conditions to other final conditions, due to the destabilization of the system. The simplest processes can be carried out on isolated single-component systems in which we can vary some of their internal bonds. Changes must be quasi-static. That is, slow enough to be considered as a succession of equilibrium states. Thermodynamic processes can be interpreted as the result of the interaction of one system with another after some bond between them has been removed, so that the systems are finally in balance (mechanical, thermal and / or material) with each other.
\end{abstract}

Keywords:

Thermodynamic process, system, equilibrium state

\section{Resumen:}

Un proceso termodinámico son los cambios de un sistema, desde unas condiciones iniciales hasta otras condiciones finales, debido a la desestabilización del sistema. Los procesos más simples los podemos realizar sobre sistemas aislados mono componentes en los que podemos variar alguna de sus ligaduras internas. Los cambios deben ser cuasiestáticos. Es decir, suficientemente lentos para ser considerados como una sucesión de estados de equilibrio. Los procesos termodinámicos pueden ser interpretados como el resultado de la interacción de un sistema con otro tras ser eliminada alguna ligadura entre ellos, de forma que finalmente los sistemas se encuentren en equilibrio (mecánico, térmico y/o material) entre sí.

\section{Palabras Clave:}

Proceso termodinámico, sistema, estado de equilibrio

\section{Introducción}

Para poder entender de mejor forma este tema de procesos termodinámicos es necesario definir y explicar un poco sobre los sistemas termodinámicos, los cuales están constituidos por cierta cantidad de materia o radiación en una región del espacio considerada para su estudio. Al hablar de una región en el espacio, se tiene que saber que está va a ser delimitada por una frontera, la cual se define como la región que separa al sistema del resto del universo físico [1]. Esta frontera está constituida por paredes, las cuales son:

-Pared rígida: es un sistema aislado mecánicamente y su volumen permanece constante

-Pared móvil: hay variación de volumen e intercambio de trabajo mecánico.

-Pared adiabática: es cuando el sistema está aislado térmicamente.

-Pared diatérmica: es cuando el sistema intercambia calor con sus alrededores.

\footnotetext{
a Oscar Daniel Ramírez Cárdenas, Universidad Autónoma del Estado de Hidalgo, https://orcid.org/0000-0002-3015-310X, Email: od50268@gmail.com

b José Fernando Vázquez Montiel, Universidad Autónoma del Estado de Hidalgo, https://orcid.org/0000-0002-0145-3250, Email: fernandovazquez8000@gmail.com

c Brando Javier Martínez Ramírez, Universidad Autónoma del Estado de Hidalgo, https://orcid.org/0000-0002-9099-6192, Email: branduchis1220@gmail.com

d Víctor David Rodríguez Caballero, Universidad Autónoma del Estado de Hidalgo, https://orcid.org/0000-0002-4805-2032, Email: davictor6564@gmail.com
} 
Procesos Termodinámicos

En física, se denomina proceso termodinámico a la evolución de determinadas magnitudes (o propiedades) propiamente termodinámicas relativas a un determinado sistema termodinámico [2]. Estas transformaciones deben transcurrir desde un estado de equilibrio inicial a otro final; es decir, que las magnitudes que sufren una variación al pasar de un estado a otro deben estar perfectamente definidas en dichos estados inicial y final. La representación gráfica de estos procesos se puede hacer mediante la utilización de diagramas $\mathrm{PV}$, en donde se refleja la presión $(P)$ en el eje de ordenadas y el volumen (V) en el eje de abscisas. En este tipo de diagrama se representa el estado de un sistema termodinámico (como un punto en el mismo) o un proceso termodinámico del sistema (como una curva en él).

Existen 4 tipos de procesos termodinámicos:

-Proceso isotérmico: es aquel durante el cual la temperatura $(T)$ permanece constante.

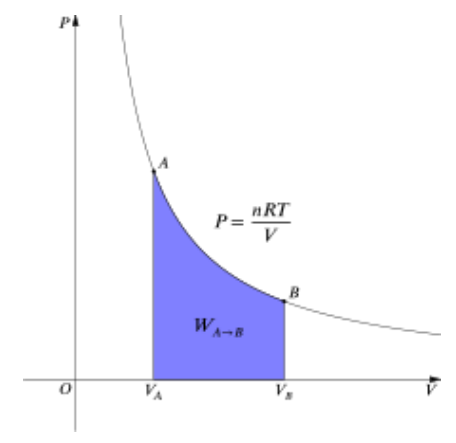

Figura 1. Proceso isotérmico representado en un diagrama $P V$.

Proceso isobárico: aquel en el que la presión $(P)$ permanece constante.

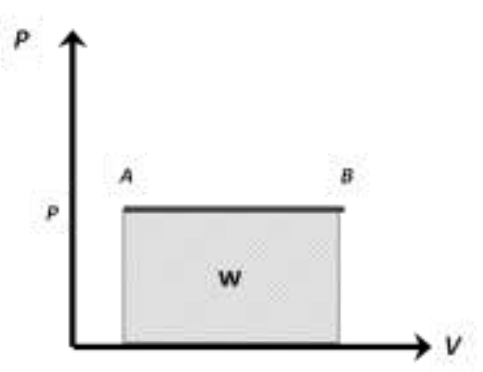

Figura 2. Proceso Isobárico representado en un diagrama PV.

Proceso isocórico o isométrico: donde el volumen específico (V) permanece constante

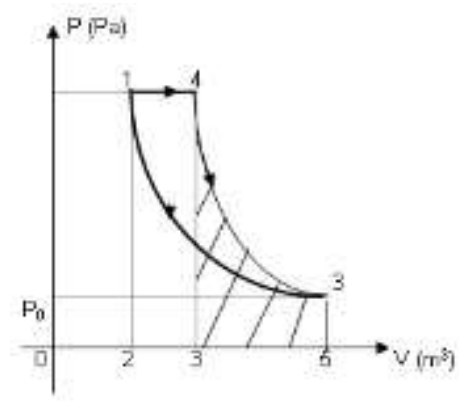

Figura 3. Proceso isocórico representado en un diagrama $P V$.

Proceso adiabático o isotrópico: donde su calor (Q) es constante [3].

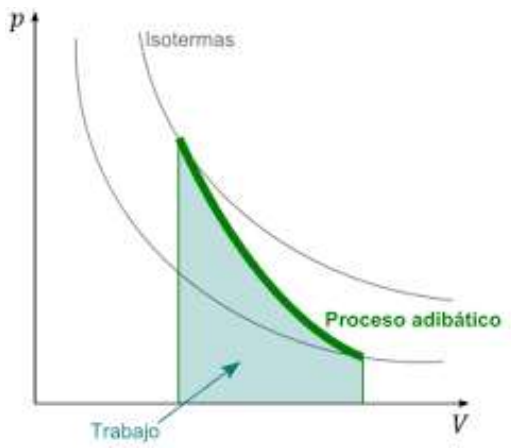

Figura 4. Proceso adiabático representado en un diagrama $P V$

\section{Primera ley de la termodinámica}

La primera ley de la termodinámica establece que, cuando se añade calor (Q) a un sistema mientras éste efectúa trabajo $(W)$, la energía interna $(\Delta U)$ cambia en una cantidad igual a $Q-W$.

La primera ley de termodinámica es la misma ley del principio de conservación de la energía, la cual exige que para todo sistema termodinámico se cumpla:

$$
\Delta U=Q-W
$$

Siendo $\Delta \boldsymbol{U}$ la energía interna del sistema [4] 


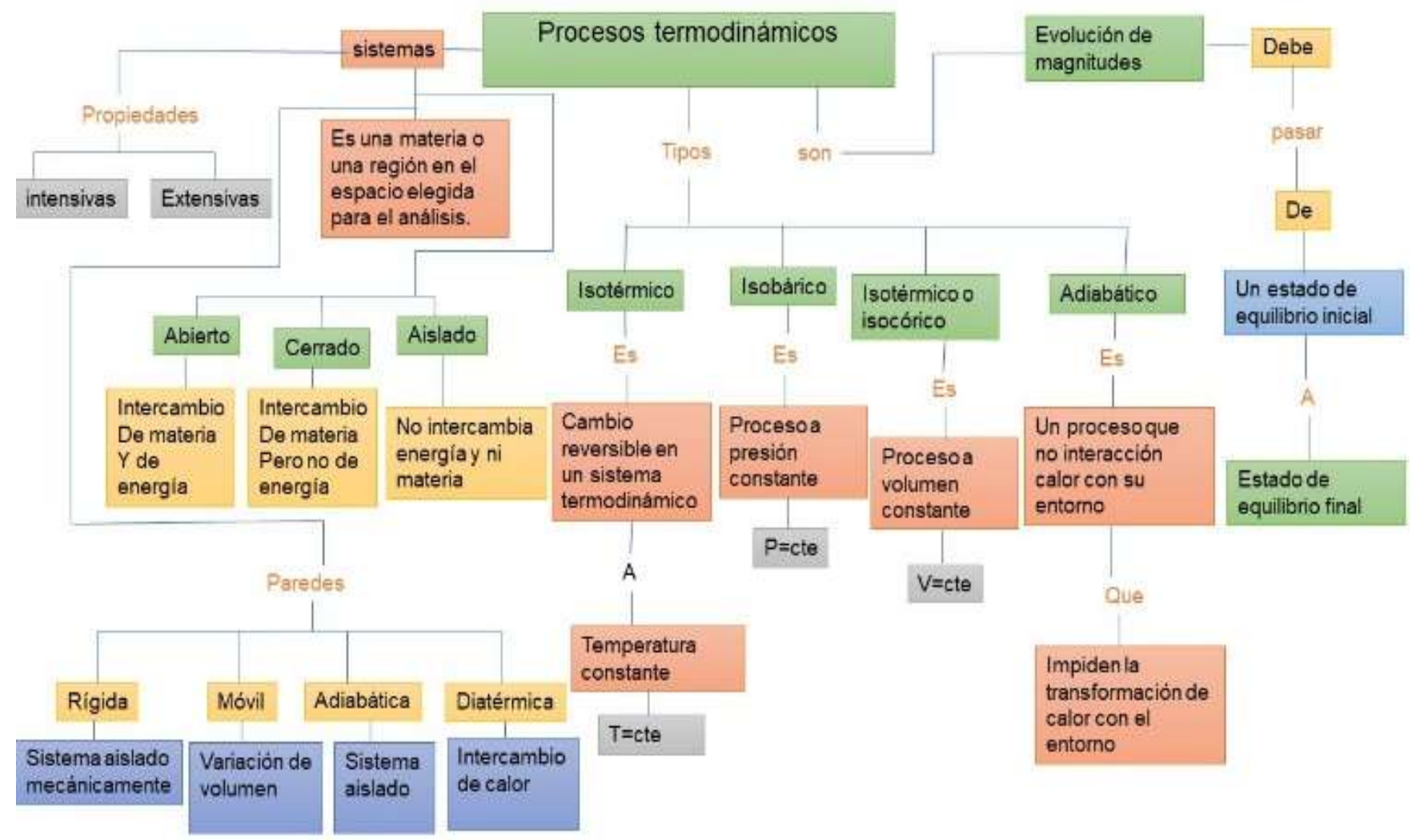

\section{Referencias}

[1] Física. (s.f.). Recuperado el 6 de Abril de 2020, de Termodinámica: https://ibero.mx/campus/publicaciones/fisica/pdf/15termodinamica.pdf

[2] Termodinámica. (s.f.). Recuperado el Abril de 2020, de Lección 6: https://ergodic.ugr.es/termo/lecciones/leccion06.pdf

[3] Física CEFA. (2009). Procesos termodinámicos, de blog Sitio web: http://fisicacefa1 1.over-blog.org/article-30040201.html

[4] Tipler, Paul A. (1999). «Capítulo 19: Calor y primer principio de la termodinámica». Física para la ciencia y la tecnología (Cuarta edición). Reverté. p. 570-573.

[5] Física. (s.f.). Recuperado el 5 de Abril de 2020, de Procesos y ciclos: http://www.fis.puc.cl/ jalfa ro/fis $1523 /$ cla ses/2\%20Procesos $\% 20 y \% 20 \mathrm{Cicl}$ os.pdf

[6] Termodinámica. (s.f.). Recuperado el Abril de 2020, de Lección 6:

https://ergodic.ugr.es/termo/lecciones/leccion06.pdf 